\title{
MODELLING OF GENERALIZED NEWTONIAN LID-DRIVEN CAVITY FLOW USING AN SPH METHOD
}

\author{
ASHKAN RAFIEE ${ }^{1}$ \\ (Received 21 May, 2007; revised 8 January, 2008)
}

\begin{abstract}
In this paper a smoothed particle hydrodynamics (SPH) method is introduced for simulating two-dimensional incompressible non-Newtonian fluid flows, and the nonNewtonian effects in the flow of a fluid which can be modelled by generalized Newtonian constitutive equations are investigated. Two viscoplastic models including Bingham-plastic and power-law models are considered along with the Newtonian model. The governing equations include the conservation of mass and momentum equations in a pseudo-compressible form. The spatial discretization of these equations is achieved by using the SPH method. The temporal discretization algorithm is a fully explicit two-step predictor-corrector scheme. In the prediction step, an intermediate velocity field is obtained using a forward scheme in time without enforcing incompressibility. The correction step consists of solving a pressure Poisson equation to satisfy incompressibility by providing a trade-off between the pressure and density variables. The performance of the proposed scheme is evaluated by studying a benchmark problem including flow of viscoplastic fluids in a lid-driven cavity. Both Newtonian and non-Newtonian cases are investigated and the results are compared with available numerical data. It was shown that in all cases the method is stable and the results are in very good agreement with available data.
\end{abstract}

2000 Mathematics subject classification: primary 76M28; secondary 76A05.

Keywords and phrases: smoothed particle method, incompressible non-Newtonian flows, generalized Newtonian constitutive equations.

\section{Introduction}

Many modern materials and manufacturing processes require the investigation of both Newtonian and non-Newtonian fluids. The categories of viscous and viscoelastic non-Newtonian fluids and the variety of related constitutive equations they provide cover applications such as petroleum, lubricants, food industry and blood flow [17]. Examples of such materials can be found in the case of solutions and melts of macromolecules. The range of behaviour is also diverse: for example, coating

\footnotetext{
${ }^{1}$ Center of Excellence in Energy Conversion, School of Mechanical Engineering, Sharif University of Technology, Tehran, Iran; e-mail: ashkan_rafiee@ mech.sharif.ir.

(C) Australian Mathematical Society 2008, Serial-fee code 0334-2700/08
} 
processes frequently involve fluids that exhibit solid-like behaviour at low shear rates and a viscous behaviour beyond a yield limit at higher shear rates. Such material properties, first described by Bingham in 1922, are the characteristics of Binghamplastic fluids [3]. These constitutive equations describe the flow of a non-Newtonian fluid in the form of a fluid with a 'shear rate' dependent viscosity. Both Bingham and power-law fluids are examples of simple non-Newtonian constitutive models considered as the 'generalized Newtonian' model. A computational fluid dynamic (CFD) approach to non-Newtonian problems is generally far more complicated than Newtonian cases owing to the complexity introduced by the constitutive equation of the utilized model in the diffusion term of the Navier-Stokes equation. A number of methods have been worked out to study non-Newtonian fluid flows [3, 13, 20, 21].

Beside the traditional Eulerian methods, such as the finite element method (FEM), an alternative class of methods which has attracted much attention is the meshless method. This is partly due to the ease of implementation and also partly because of the independence from grid information. In fact, the word 'meshless' means that in these methods there is no inherent reliance on a particular mesh topology. In practice, however, in many meshless methods recourse must be taken to some kind of background meshes at least in one stage of the implementation.

The smoothed particle hydrodynamics (SPH) method is a meshless technique which was originally developed in 1977 by Lucy [12] and Gingold and Monaghan [10]. The method uses a purely Lagrangian approach and has been successfully employed in a wide range of problems. The SPH method is a particle-based method; here, however, the word 'particle' does not refer to a physical mass of fluid, but rather to a region in space. Field variables are associated to these particles and at any other point in space are found by averaging, or smoothing, the particle values over the region of interest. This is fulfilled by an interpolation or weight function which is often called the interpolation kernel.

The SPH method was originally developed to solve compressible astrophysical problems [10, 12]. The method was later extended to incompressible flows by Monaghan [15] and Takeda et al. [19] and simulating non-Newtonian flows with SPH attracted much research. Ellero et al. developed a numerical scheme based on the SPH method to study viscoelastic fluid flows using a Maxwell model [6, 7]. Also, Shao et al. presented an SPH method to solve non-Newtonian flow with free surfaces using a modified form of the so-called cross model [18].

The motivation of this work is to present the ability of the SPH method to simulate non-Newtonian fluid flows which can be modelled by generalized Newtonian models. The performance of the proposed solution algorithm is demonstrated by solving the famous benchmark problem known as lid-driven cavity flow for both Bingham and power-law fluids along with a Newtonian fluid.

\section{Governing equations}

2.1. Dimensional form The governing equations for simulating fluid flow in two dimensions are the mass and momentum conservation equations. The Lagrangian form 
of these equations can be written as

$$
\begin{gathered}
\frac{1}{\rho} \frac{D \rho}{D t}+\nabla \cdot \mathbf{U}=0, \\
\frac{D \mathbf{U}}{D t}=\mathbf{g}+\frac{1}{\rho} \nabla \cdot \boldsymbol{\tau}-\frac{1}{\rho} \nabla P,
\end{gathered}
$$

where $\rho$ is density, $t$ is time, $\mathbf{U}$ is the velocity vector, $P$ is pressure, $\mathbf{g}$ is gravitational acceleration and $\tau$ is shear stress tensor. In the above two equations, $D / D t$ is the material (total) derivative defined in the fixed Eulerian frame by

$$
D / D t=\partial / \partial t+\mathbf{U} \cdot \nabla
$$

In order to close the system of Equations (2.1) and (2.2) we require constitutive equations for $P$ and $\tau$. For an incompressible generalized Newtonian fluid the extra stress tensor reduces to a shear stress tensor with a viscosity $\eta$ that is a function of the second scalar invariant of the rate-of-strain tensor $\mathbf{D}$ :

$$
\begin{aligned}
\tau & =2 \eta(|\mathbf{D}|) \mathbf{D}, \\
\mathbf{D} & =\frac{1}{2}\left(\nabla \mathbf{U}+\nabla \mathbf{U}^{\mathrm{T}}\right) .
\end{aligned}
$$

For a Newtonian fluid the viscosity is constant for a given temperature, pressure and composition. For the power-law model, it is given that the viscosity is a function of shear rate as follows [2]:

$$
\eta=m|\mathbf{D}|^{n-1}
$$

The power-law model contains two parameters, $m$ ( $\left.\mathrm{Pa} \mathrm{s}^{-1}\right)$ and $n$ (dimensionless). If $n<1$, the fluid is said to be pseudo-plastic or shear thinning; and if $n>1$, the fluid is called dilatant or shear thickening [2]. For the Bingham model, $\eta$ is given by [2]

$$
\left\{\begin{array}{l}
\eta=\infty \\
\eta=\mu_{0}+\tau_{0} /(|\mathbf{D}|) .
\end{array}\right.
$$

In this model, $\mu_{0}$ represents the Newtonian viscosity and $\tau_{0}$ is the yield stress, for stresses below which the fluid behaves like a rigid solid.

2.2. Dimensionless forms and scaling A convenient form of the governing equations can be obtained by defining the following nondimensional variables:

$$
\begin{aligned}
& x=\frac{x^{*}}{L_{*}}, \quad y=\frac{y^{*}}{L}, \quad u=\frac{u^{*}}{U}, \quad v=\frac{v^{*}}{U}, \\
& \rho=\frac{\rho^{*}}{\rho_{0}}, \quad p=\frac{P}{\rho_{0} U^{2}} \quad \text { and } \quad \tau=\frac{\tau^{*}}{\rho_{0} U^{2}} .
\end{aligned}
$$


Here, the superscript $*$ denotes dimensional variables. The nondimensional system of governing equations is then written as

$$
\begin{aligned}
\frac{D \rho^{*}}{D t^{*}} & =-\rho^{*} \frac{\partial u_{i}^{*}}{\partial x_{j}^{*}}, \\
\frac{D u_{i}^{*}}{D t^{*}} & =\frac{g_{i}^{*}}{F r^{2}}+\frac{1}{\rho^{*}} \frac{\partial \tau_{i j}^{*}}{\partial x_{j}^{*}}-\frac{1}{\rho^{*}} \frac{\partial P^{*}}{\partial x_{i}^{*}}, \\
\tau^{*} & =\frac{1}{R e} 2 \eta^{*} \mathbf{D}^{*} .
\end{aligned}
$$

The dimensionless number $R e$ that appears in the above equations is the Reynolds number,

$$
R e=\frac{\rho_{0} U_{0} L_{0}}{\eta_{0}}
$$

\section{SPH formulation}

3.1. Basic concepts The SPH method is based on the interpolation theory. The method allows any function to be expressed in terms of its values at a set of disordered points, the particles. If we consider $A(r)$ as a typical field variable at a certain position, $r$, in space, the kernel estimate of $A(r)$ is defined as [14]:

$$
\left\langle A_{h}(\mathbf{r})\right\rangle=\int_{\text {space }} A\left(\mathbf{r}^{\prime}\right) W\left(\mathbf{r}-\mathbf{r}^{\prime}, h\right) d \mathbf{r}^{\prime} .
$$

Here $h$ represents the smoothing length and $W$ is a weighting function with the following properties:

$$
\int_{\text {space }} W\left(\mathbf{r}-\mathbf{r}^{\prime}, h\right) d \mathbf{r}^{\prime}=1, \quad \lim _{h \rightarrow 0} W\left(\mathbf{r}-\mathbf{r}^{\prime}, h\right)=\delta\left(\mathbf{r}-\mathbf{r}^{\prime}\right) .
$$

Eventually, Equation (3.1) can be expressed as

$$
\left\langle A_{h}(\mathbf{r})\right\rangle=\sum_{j=1}^{N} \frac{m_{j}}{\rho_{j}} A_{j} W\left(\mathbf{r}-\mathbf{r}_{j}, h\right),
$$

where the summation index $j$ denotes a particle label, and $m_{j}, \rho_{j}$ and $A_{j}$ are the particle mass, density and field variable, respectively.

3.2. Kernel function The kernel function is the most important ingredient of the SPH method. Various forms of kernels with different compact support have been proposed by many researchers. Recent studies $[14,16]$ indicate that the stability of the SPH algorithm depends strongly upon the second derivative of the kernel. In this 
work, initially a cubic spline kernel was used. It was found however that the quintic spline kernel leads to more accurate results. The quintic spline kernel is defined as [7]

$$
W(r, h)=W_{0} \times \begin{cases}(3-s)^{5}-6(2-s)^{5}+15(1-s)^{5}, & 0 \leq s<1, \\ (3-s)^{5}-6(2-s)^{5}, & 1 \leq s<2, \\ (3-s)^{5}, & 2 \leq s<3, \\ 0, & 3 \leq s,\end{cases}
$$

where in $2 \mathrm{D}$ the normalization factor $W_{0}$ takes the value $7 /\left(478 \pi h^{2}\right)$. This kernel has the advantage that its first four derivatives are continuous and has a compact support $3 h$.

3.3. Gradient/divergence The gradient and divergence need to be formulated in an $\mathrm{SPH}$ algorithm if simulation of the Navier-Stokes equation is to be attempted. There are a number of different ways of representing these operators. In this work, the following approximations are used [4]:

$$
\begin{aligned}
\frac{1}{\rho_{i}} \nabla_{i} A & =\sum_{j} m_{j}\left(\frac{A_{i}}{\rho_{i}^{2}}+\frac{A_{j}}{\rho_{j}^{2}}\right) \nabla_{i} W_{i j}, \\
\frac{1}{\rho_{i}} \nabla_{i} \cdot \mathbf{U}_{i} & =\sum_{j} m_{j}\left(\frac{\mathbf{U}_{i}}{\rho_{i}^{2}}+\frac{\mathbf{U}_{j}}{\rho_{j}^{2}}\right) \cdot \nabla_{i} W_{i j},
\end{aligned}
$$

where $\nabla_{i} W_{i j}$ denotes the gradient of the kernel function $W\left(\left|\mathbf{r}_{i}-\mathbf{r}_{j}\right|, h\right)$ with respect to $\mathbf{r}_{i}$. There are a number of diverse ways of representing these operators but this choice of discretization operators ensures that an exact projection algorithm is produced.

3.4. Laplacian A simple way to formulate the Laplacian operator is to envisage it as the dot product of the divergence and gradient operators. In this paper, the following alternative approach is adopted [5]:

$$
\nabla \cdot\left(\frac{1}{\rho} \nabla A\right)_{i}=\sum_{j} m_{j} \frac{8}{\left(\rho_{i}+\rho_{j}\right)^{2}} \frac{A_{i j} \mathbf{r}_{i j} \cdot \nabla_{i} W_{i j}}{\left|\mathbf{r}_{i j}^{2}\right|+\epsilon^{2}}
$$

where $A_{i j}=A_{i}-A_{j}, \mathbf{r}_{i j}=\mathbf{r}_{i}-\mathbf{r}_{j}$ and $\epsilon$ is a small number introduced to avoid a zero denominator during computations and is set to $0.1 \mathrm{~h}$.

3.5. Viscous terms Several forms of viscosity were presented for the SPH method by Lucy [12] and Gingold and Monaghan [10]. In this work, the divergence of the stress tensor in the momentum equation is represented by

$$
\left(\frac{1}{\rho} \nabla \cdot \boldsymbol{\tau}\right)_{i}=\sum_{j} m_{j}\left(\frac{\boldsymbol{\tau}_{i}}{\rho_{i}^{2}}+\frac{\boldsymbol{\tau}_{j}}{\rho_{j}^{2}}\right) \cdot \nabla_{i} W\left(\mathbf{r}_{i j}, h\right),
$$

where in two dimensions

$$
\nabla_{i} W\left(\mathbf{r}_{i j}, h\right)=\frac{d W}{d \mathbf{r}_{i j}} \frac{1}{\left|\mathbf{r}_{i j}\right|}\left[\left(x_{i}-x_{j}\right) \mathbf{i}+\left(y_{i}-y_{j}\right) \mathbf{j}\right] .
$$


Typical derivatives needed for Equation (2.5) can be evaluated in the SPH context as

$$
\begin{aligned}
& \left(\frac{\partial u}{\partial x}\right)_{i}=\sum_{j} \frac{m_{j}}{\rho_{j}}\left(u_{j}-u_{i}\right) \frac{x_{i}-x_{j}}{\left|\mathbf{r}_{i j}\right|} \frac{d W}{d \mathbf{r}_{i j}}, \\
& \left(\frac{\partial u}{\partial y}\right)_{i}=\sum_{j} \frac{m_{j}}{\rho_{j}}\left(u_{j}-u_{i}\right) \frac{y_{i}-y_{j}}{\left|\mathbf{r}_{i j}\right|} \frac{d W}{d \mathbf{r}_{i j}},
\end{aligned}
$$

where $\mathbf{r}_{i j}=\mathbf{r}_{i}-\mathbf{r}_{j}$. Other derivatives can be calculated in the same fashion.

\section{Simulating the boundary}

Simulating boundaries in SPH has received special attention during the past decades. A number of strategies have been implemented. The employed approach for simulating a boundary in this paper is the Morris model [16]. Morris used special particles designed so that they fill the boundary region uniformly. The boundary particles contribute to the density such that the pressure decreases when the fluid and the boundary particle diverge. The problem is that the true velocity of the boundary particle is always zero so they cannot interact correctly with free particles. Morris came up with a way that assigns an artificial velocity to a boundary particle. In this case for each free particle 'A' the normal distance $d_{\mathrm{A}}$ to the boundary is calculated, and this is used to evaluate the tangent line according to the normal of ' $\mathrm{A}$ '. Then the normal distance $d_{\mathrm{B}}$ from boundary particle 'B' to the tangent line has to be calculated. Eventually the artificial velocity for the boundary particle is given by

$$
U_{\mathrm{B}}=-\frac{d_{\mathrm{B}}}{d_{\mathrm{A}}} U_{\mathrm{A}} .
$$

\section{Solution procedure}

As mentioned before, in this work a two-step prediction-correction scheme is used to march in time. Figure 1 shows the solution procedure that we employed in this paper.

The first prediction step is integration forward in time regardless of incompressibility and so the temporal particle velocity and position are obtained as [19]

$$
\begin{aligned}
(\triangle \mathbf{U})^{*} & =\left(\mathbf{g}+\frac{1}{\rho} \nabla \cdot \tau\right) \triangle t, \\
\mathbf{U}^{*} & =\mathbf{U}_{t}+\triangle \mathbf{U}^{*}, \\
\mathbf{r}^{*} & =\mathbf{r}_{t}+\mathbf{U}^{*} \Delta t,
\end{aligned}
$$

where $\mathbf{U}_{t}$ and $\mathbf{r}_{t}$ are particle velocity and position at time $t$ and likewise superscript $*$ denotes the temporal field variable. Because we did not enforce incompressibility in the first step the density of the fluid deviates from the initial density $\rho_{0}$ and hence we use the symbol $\rho^{*}$ for it. 


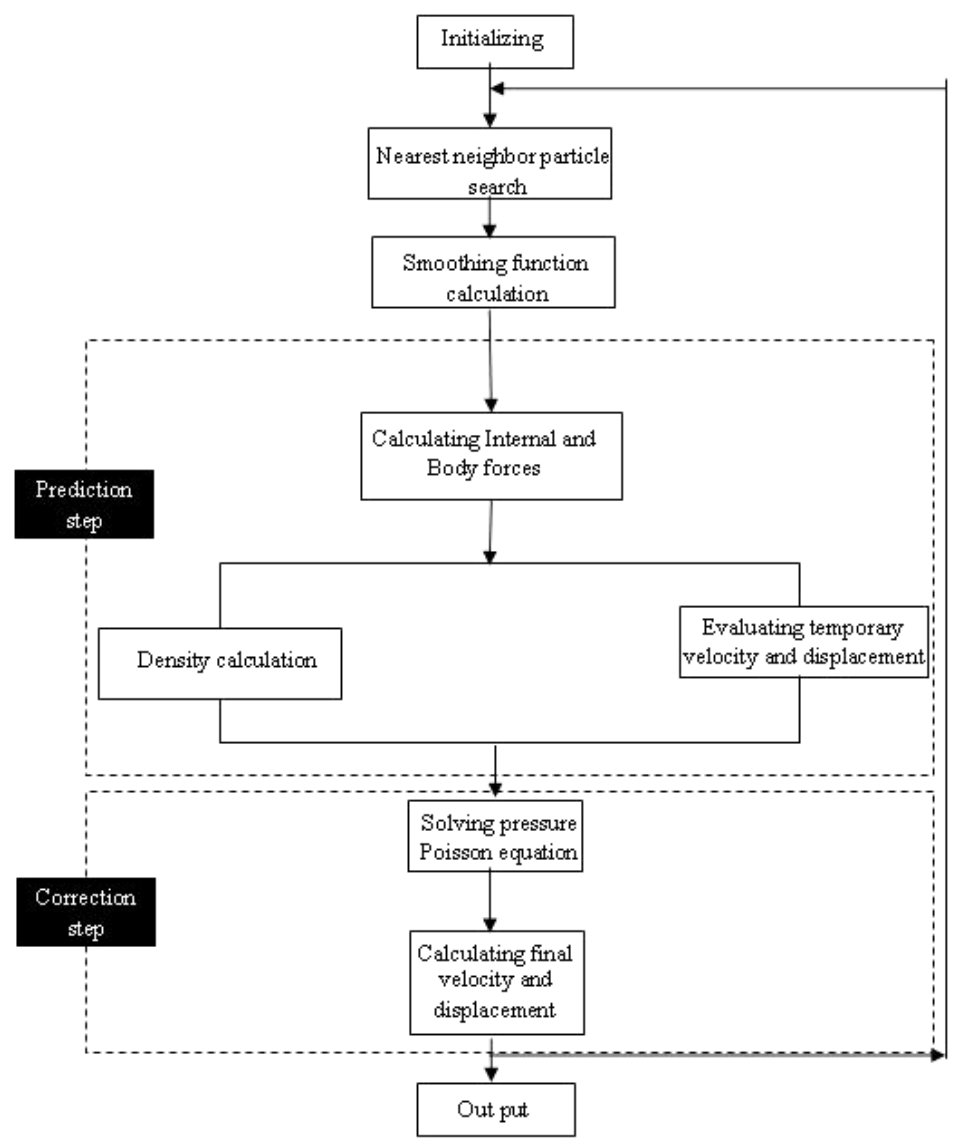

FIGURE 1. Solution procedure.

In the second and prediction step we solve the pressure Poisson equation to enforce incompressibility [11]. Thus the relevant steps are

$$
\begin{aligned}
\nabla \cdot\left(\frac{\nabla P_{t+\Delta t}}{\rho^{*}}\right) & =\frac{\rho_{0}-\rho^{*}}{\rho_{0} \Delta t^{2}}, \\
(\triangle \mathbf{U})^{* *} & =\frac{-1}{\rho^{*}} \nabla P_{t+\Delta t} \Delta t, \\
\mathbf{U}_{t+\Delta t} & =\mathbf{U}^{*}+\Delta \mathbf{U}^{* *}, \\
\mathbf{r}_{t+\Delta t} & =\mathbf{r}_{t}+\frac{U_{t}+U_{t+\Delta t}}{2} \Delta t .
\end{aligned}
$$

Finally, at the end of each round of computations, the initial density of the fluid is restored. For numerical stability, several time step constraints must be satisfied as 


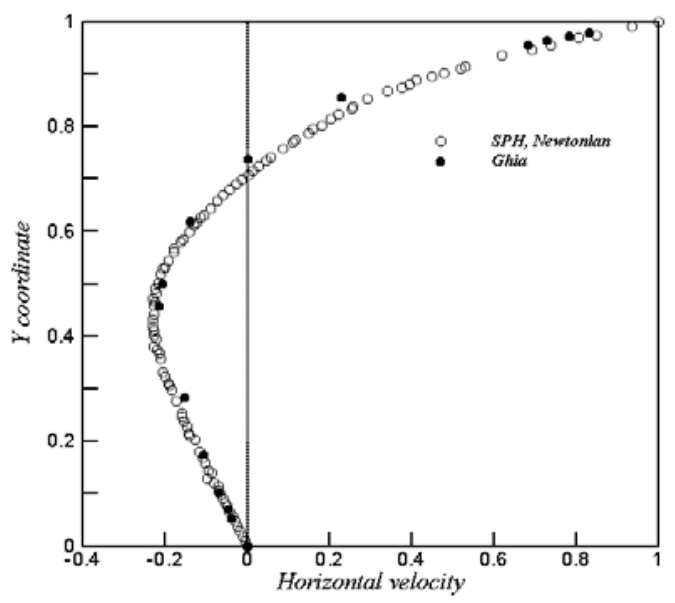

FIGURE 2. U-velocity profile along vertical centreline for Newtonian fluid.

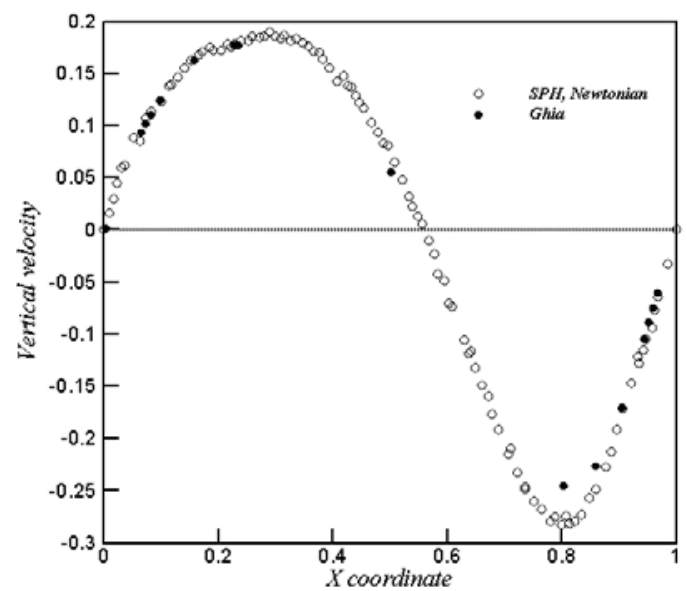

FIGURE 3. V-velocity profile along horizontal centreline for Newtonian fluid.

Courant-Friedrichs-Lewy (CFL) and viscous diffusion conditions:

$$
\begin{aligned}
& \Delta t \leq 0.1 \frac{l_{0}}{U_{\max }}, \\
& \Delta t \leq 0.5 \frac{l^{2}}{\mu / \rho} .
\end{aligned}
$$




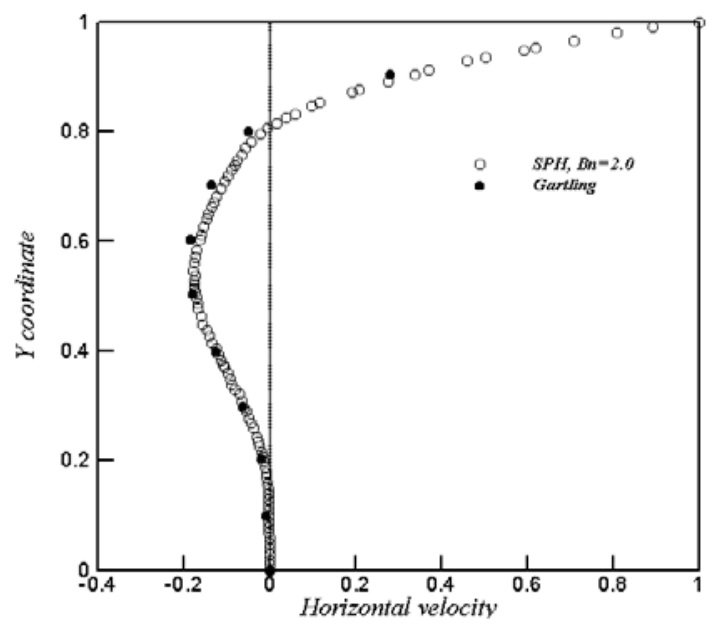

FIGURE 4. U-velocity profile along vertical centreline for Bingham fluid.

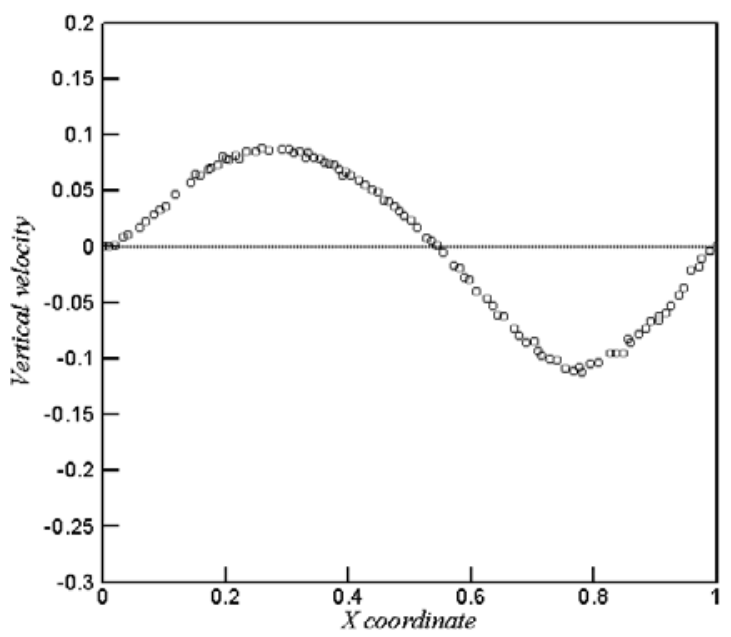

FIGURE 5. V-velocity profile along horizontal centreline for Bingham fluid.

In (5.8), $U_{\max }$ is the maximum of velocity in each time step and $l_{0}$ is the initial particle spacing; and (5.9) comes from the usual condition for an explicit finite difference method for simulating diffusion.

\section{The lid-driven cavity flow}

The lid-driven cavity problem has been used to demonstrate the capability of the SPH method to simulate fluid flows in the presence of both moving and fixed boundaries. The Reynolds number considered was $R e=100$ for both Newtonian and 


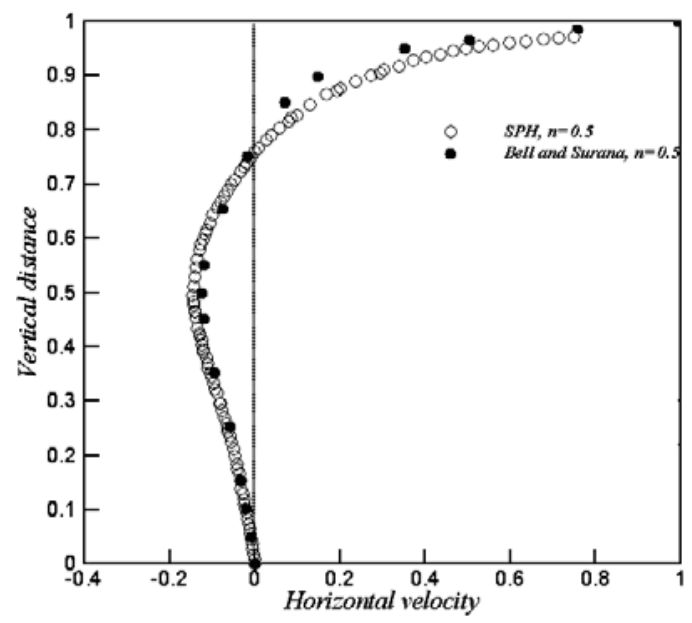

FIGURE 6. U-velocity profile along vertical centreline for power-law fluid.

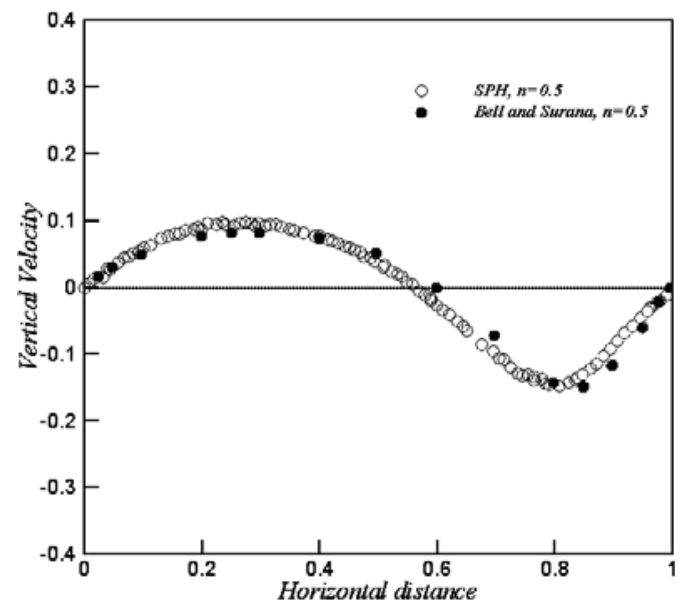

FIGURE 7. V-velocity profile along horizontal centreline for power-law fluid.

generalized Newtonian cases (Bingham and power-law fluids) and in all simulations 10000 particles are used (regardless of boundary particles). For the Bingham model the following parameters were considered:

$$
\alpha=\frac{\eta}{\eta_{r}}=0.01, \quad N_{\mathrm{B}}=\frac{\tau_{0} L}{\eta U_{0}}=2,
$$

where $N_{\mathrm{B}}$ is the Bingham number, $\eta$ and $\eta_{r}$ are Newtonian and pre-yield viscosities, respectively. 
The simulation for the power-law model is performed with $n=0.5$ in (2.6), which shows the behaviour of 'pseudo-plastic' or 'shear thinning' fluids and parameter $m$ was set to be 1 .

For validating the Newtonian results the results of Ghia et al. [9] are used; in the case of a non-Newtonian fluid for the Bingham model those of Gartling [8] are used; while for a power-law model the results obtained by Bell and Surana [1] are employed.

Since both normal and shear stresses are small in the lower half of the cavity, in the Bingham model this part of the cavity behaves as a perfect rigid solid. Consequently the centre of the main vortex in the Bingham fluid is placed at a higher height compared with the Newtonian fluid. The results shown in the figures demonstrate a very good agreement in comparison with available data.

For comparison of the flow of a non-Newtonian fluid with a Newtonian fluid at the same Reynolds number, we can see that the behaviour of the fluids in the lower portion of the cavity is markedly different. Another significant difference is the position of the central recirculating zone in Newtonian and non-Newtonian fluids, which also differs in the Bingham and power-law models.

\section{Conclusion}

A numerical code for the computation of viscous non-Newtonian flows has been presented. The code is based upon the prediction-correction SPH method. NonNewtonian flow computations were performed using Bingham and power-law models. The code has been validated against both Newtonian and non-Newtonian lid-driven cavity flow cases. It was demonstrated that this method has a strong capability in modelling the flows in the presence of moving and stationary boundaries and also with very good accuracy can model the vortices in the flow. Current results were in very good agreement with results by previous researchers for both Newtonian and nonNewtonian fluid flow cases.

\section{References}

[1] B. C. Bell and K. S. Surana, " $p$-version least squares finite element formulation for twodimensional, incompressible, non-Newtonian, isothermal and non-isothermal flow”, Internat. J. Numer. Methods Fluids 18 (1994) 127-162.

[2] R. B. Bird, R. C. Armstrong and O. Hassager, Dynamics of polymeric liquids-fluid mechanics, 2nd edn (Wiley-Interscience, New York, 1987).

[3] A. Bose and G. F. Carrey, "Least-square $p-r$ finite element methods for incompressible nonNewtonian flows", J. Comput. Methods Appl. Engrg. 180 (1999) 431-458.

[4] A. Colagrossi and M. Landrini, "Numerical simulation of interfacial flows using smoothed particle hydrodynamics", J. Comput. Phys. 191 (2003) 448-475.

[5] S. J. Cummins and M. Rudman, "An SPH projection method”, J. Comput. Phys. 152 (1999) 584607.

[6] M. Ellero, M. Kroger and S. Hess, "Viscoelastic flows studied by smoothed particle hydrodynamics", J. Non-Newtonian Fluid Mech. 105 (2002) 35-51.

[7] M. Ellero and R. I. Tanner, "SPH simulation of transient viscoelastic flows at low Reynolds number”, J. Non-Newtonian Fluid Mech. 132 (2005) 61-72. 
[8] D. K. Gartling, "Finite element methods of non-Newtonian flows", Sandia Report SAND 92-0886, UC-705, 1992.

[9] U. Ghia, K. N. Ghia and C. T. Shin, "High-resolution for incompressible flow using Navier-Stokes equations and a multigrid method", J. Comput. Phys. 481 (1982) 387-411.

[10] R. A. Gingold and J. J. Monaghan, "Smoothed particle hydrodynamics: theory and application to nonspherical stars", Mon. Not. Roy. Astr. Soc. 181 (1977) 275-389.

[11] S. M. Hosseini, M. T. Manzari and S. K. Hannani, "A fully explicit three-step SPH algorithm for simulation of non-Newtonian fluid flow", Internat. J. Numer. Methods Heat Fluid Flow 17 (2007) 715-735.

[12] L. B. Lucy, "A numerical approach to the testing of fission hypothesis", Astron. J. 82 (1977) 10131020.

[13] H. Matallah, P. Townsend and M. F. Webster, "Viscoelastic computations of polymeric wirecoating flows", Internat. J. Numer. Methods. Heat Fluid Flow 12 (2002) 404-433.

[14] J. J. Monaghan, "Smoothed particle hydrodynamics", Annu. Rev. Astron. Astrophys. 30 (1992) $543-574$.

[15] J. J. Monaghan, "Simulating free surface flows with SPH”, J. Comput. Phys. 110 (1994) 399-406.

[16] J. P. Morris, P. J. Fox and Y. Zhu, "Modelling low Reynolds number incompressible flows using SPH”, J. Comput. Phys. 136 (1997) 214-226.

[17] P. Neofytou, "A 3rd order upwind finite volume method for generalized Newtonian fluid flows", Adv. Engrg. Software 36 (2005) 664-680.

[18] S. Shao and E. Y. M. Lo, "Incompressible SPH method for simulating Newtonian and nonNewtonian flows with a free surface", Adv. Water Resources 26 (2003) 787-800.

[19] H. Takeda, S. M. Miyama and M. Sekiya, "Numerical simulation of viscous flow by smoothed particle hydrodynamics", Prog. Theor. Phys. 92 (1994) 939-960.

[20] D. Vola, F. Babik and J. C. Latche, "On a numerical strategy to compute gravity currents of nonNewtonian fluids", J. Comput. Phys. 201 (2004) 397-420.

[21] M. F. Webster, H. R. Tamaddon-Jahromi and M. Aboubacar, "Transient viscoelastic flow in planar contractions", J. Non-Newtonian Fluid Mech. 118 (2004) 83-101. 